\title{
Dialisi peritoneale in Campania: marginale... o emarginata?
}

\author{
Bruno Memoli ${ }^{1}$, Gennaro Argentino ${ }^{2}$, Roberta Russo ${ }^{2}$, Immacolata Daniela Maresca ${ }^{2}$ \\ ${ }^{1}$ Direttore dell'UOC di Tecniche Emodialitiche e Dialisi Peritoneale, Università degli Studi di Napoli Federico II, \\ Napoli \\ ${ }^{2}$ DAI di Chirurgie Specialistiche, Nefrologia, Università degli Studi di Napoli Federico II, Napoli
}

\begin{abstract}
Peritoneal dialysis in Campania region: marginal or...marginalized?
Abstract. Despite its clinical and economic benefits and its ability to improve quality of life, peritoneal dialysis is still not proposed or performed in Campania region ( $<2 \%$ of cases), thus leading to higher health care costs. Our centre, the first in Campania region, currently numbers 52 patients.
\end{abstract}

Key words: Peritoneal dialysis, Health care spending, Campania region

Conflict of interest: None.

Financial support: None.

Accettato: 24 Luglio 2013

Parafrasando Stanley Shaldon (1998), in Campania la Dialisi Peritoneale viene solitamente considerata "una metodica di seconda scelta, per medici di seconda scelta, per pazienti di seconda scelta". La massiccia presenza del privato accreditato e un insieme di pregiudizi e false convinzioni (disinteressate e non) penalizzano, anche nelle nuove generazioni di nefrologi, la diffusione e lo sviluppo di questa eccellente tecnica sostitutiva.

Le regole di buona pratica clinica nonché la carta dei diritti del paziente nefropatico imporrebbero, al momento di iniziare il trattamento sostitutivo, una dettagliata informazione del paziente circa l'esistenza, i vantaggi e gli svantaggi delle 2 diverse tipologie di trattamento dialitico (nonché delle opzioni di trapianto pre-emptive e da vivente) (1). Dopo essere stato correttamente informato, il paziente dovrebbe essere, quindi, libero di scegliere quale trattamento praticare (fatta eccezione per i pochi casi in cui la scelta è obbligata dall'esistenza di controindicazioni assolute verso l'uno o l'altro tipo di trattamento dialitico). Nella nostra Regione, sfortunatamente, tale comportamento virtuoso resta solitamente disatteso e, nella maggior parte dei casi, al paziente viene presentato e offerto un solo tipo di trattamento dialitico, quello extracorporeo, quasi sempre in un Centro privato accreditato. Frequentemente, infatti, incontriamo pazienti in trattamento emodialitico che ignorano del tutto l'esistenza della Dialisi Peritoneale fino a quando questo trattamento sostitutivo non viene loro proposto come ultima spiaggia per l'esaurimento completo del patrimonio vascolare disponibile per il confezionamento dell'accesso per l'emodialisi.

In Italia, la prevalenza della Dialisi Peritoneale è, in media, pari al 10\% circa della popolazione dialitica (in alcune Regioni è anche superiore). Dai dati raccolti nel Registro Regionale, si evince, invece, che in Campania la prevalenza della Dialisi Peritoneale è pari al $2 \%$ circa (in pratica, su circa 5500 pazienti, poco più di un centinaio pratica la Dialisi Peritoneale). Tale bassissima percentuale è probabilmente dovuta anche al fatto che 1 ' $85-90 \%$ dei trattamenti emodialitici si effettua nei Centri privati accreditati, dove la Dialisi Peritoneale non viene praticata in quanto non autorizzata. La Campania conta 136 Centri di dialisi extracorporea (dati del 2011), di cui 108 sono privati accreditati e solo 28 sono ospedalieri; di questi, soltanto la metà (14 Centri pubblici) pratica la Dialisi Peritoneale!

Questi scoraggianti numeri pongono la nostra Regione agli ultimissimi posti rispetto alla media nazionale, in barba ai vantaggi clinici della Dialisi Peritoneale ma anche a una politica di contenimento dei costi della spesa sanitaria regionale. In questo momento di grave crisi economica e di spending review, infatti, come non pensare al minore impatto economico della Dialisi Peritoneale sul budget sanitario? Tale metodica, infatti, richiede, in Campania (dove non è previsto, a tutt'oggi, un rimborso regionale per il trattamento), il solo costo del materiale, che va dai 30 ai 40 euro/die (a seconda delle metodiche 


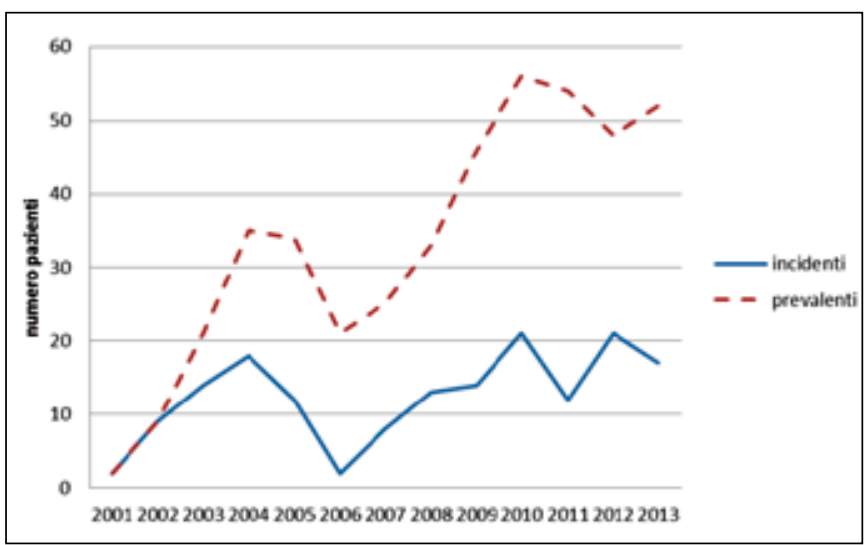

Fig. 1 - Andamento di prevalenza (linea tratteggiata) e di incidenza (linea continua) dei pazienti in Dialisi Peritoneale presso il nostro Centro dalla nascita (2001) a oggi (30 Luglio 2013).

e delle marche presenti in commercio). I costi del trattamento dialitico peritoneale rappresentano, pertanto, circa la metà di quelli dell'emodialisi praticata nei Centri privati (ai quali vanno, peraltro, aggiunti i rimborsi del trasporto dei pazienti dal domicilio al Centro e viceversa).

Il nostro Centro di Dialisi Peritoneale (che fa parte di una UOC di "Tecniche Emodialitiche e Dialisi Peritoneale" dell'Azienda Ospedaliero-Universitaria "Federico II" di Napoli) è attivo dal 2001. Dalla sua apertura, il Centro ha immesso in Dialisi Peritoneale, a oggi, oltre 160 pazienti, con una prevalenza media annua progressivamente crescente e un'incidenza che ha quasi sempre superato i 20 pazienti/anno (Fig. 1). Negli ultimi anni, seguiamo un numero di pazienti sempre superiore ai 40 (al 30 Luglio 2013 sono 52). Tali numeri pongono il nostro Centro al primo posto assoluto in Campania (dove sono presenti altri 13 Centri con un numero di pazienti, nella quasi totalità dei casi, inferiore a 10) e in ottima posizione anche in Italia.

Riteniamo che realizzare un Centro di Dialisi Peritoneale con un numero di pazienti $>20$ rivesta grande importanza per imparare a gestire nel migliore dei modi tutte le problematiche e le complicanze (fortunatamente molto più rare che in passato) del trattamento. A questo proposito, riteniamo indispensabile un investimento sia in termini di risorse umane (il personale va preparato e motivato!) che di spazi dedicati.

La nostra pluriennale esperienza ci permette di affermare che la Dialisi Peritoneale risulta decisamente conveniente sia in termini di migliore qualità della vita del paziente che in termini economici. $\mathrm{Ci}$ auguriamo che tali conclusioni, a cui sono arrivati moltissimi nefrologi italiani, vengano recepite anche dai nostri politici regionali che, con opportuni interventi, possano favorirne la diffusione, come è stato fatto in altre Regio- ni. Prendendo esempio dalle Regioni Piemonte e Sicilia (dove, nonostante il privato, la Dialisi Peritoneale è decisamente più praticata che in Campania), si potrebbe anche incentivarla, stanziando un rimborso per i pazienti che la scelgono (o per $\mathrm{i}$ caregiver).

Concludendo (e parafrasando Roberto Dell'Aquila) siamo tra i pochi in Campania che possono orgogliosamente e senza alcun dubbio affermare che la Dialisi Peritoneale è "una metodica di prima scelta per medici competenti e per tutti i pazienti (e sono la quasi totalità!) per i quali è consigliabile".

\section{Riassunto}

La Dialisi Peritoneale, nonostante i suoi indiscutibili vantaggi clinici, economici e di qualità della vita, continua a essere molto poco proposta e praticata nella Regione Campania (in meno del $2 \%$ dei casi). Il nostro Centro costituisce un'eccezione e conta, a oggi, 52 pazienti.

Parole chiave: Dialisi peritoneale, Spesa sanitaria, Regione Campania

Dichiarazione di conflitto di interessi: Gli Autori dichiarano di non avere conflitto di interessi.

Contributi economici degli autori: Gli Autori dichiarano di non aver ricevuto sponsorizzazioni economiche per la preparazione dell'articolo.

Indirizzo degli Autori:

Prof. Bruno Memoli

Direttore dell'UOC di Tecniche

Emodialitiche e Dialisi Peritoneale

Università degli Studi di Napoli Federico II

Via Sergio Pansini 5

80131 Napoli

bruno.memoli@unina.it

\section{Bibliografia}

1. Andreucci VE, Kerr DN, Kopple JD. Rights of chronic renal failure patients undergoing chronic dialysis therapy. Nephrol Dial Transplant 2004; 19 (1): 30-8. 